\title{
ON LEFT-TRUNCATING AND MIXING POISSON DISTRIBUTIONS
}

\author{
JORDI VALERO, MARTA PÉREZ-CASANY AND JOSEP GINEBRA
}

\begin{abstract}
The distributions obtained by left-truncating at $k$ a mixed Poisson distribution, $k \mathrm{~T}$-MP, those obtained by mixing previously left-truncated Poisson distributions, M- $k \mathrm{TP}$; and those obtained by left-truncating at $k$ a mixture of previously left-truncated Poisson distributions, $k \mathrm{~T}-\mathrm{M}-i \mathrm{TP}$, are characterized by means of their probability generating function. The $k \mathrm{~T}-\mathrm{MP}$ models are useful because they aproximate well the mechanism behind many count data generating processes, and because under the hypothesis that the mixing distribution has probability zero at zero, as in the continuous case, they allow one to estimate the first $k+1$ probabilities of the untruncated mixed Poisson model. Consequences of the characterizations obtained are that every $k \mathrm{~T}-\mathrm{MP}$ distribution is a M- $k \mathrm{TP}$ distribution but not the other way around, and that the set of distributions $k \mathrm{~T}-\mathrm{M}-i \mathrm{TP}$ is included in the set $k \mathrm{~T}-\mathrm{M}-(i+1) \mathrm{TP}$. Based on the characterizations obtained it follows that the factorial size-biased version of order $k+1$ of a mixed Poisson random variable and, under a certain condition, its shifted version of order $k+1$ are neither $k \mathrm{~T}$-MP nor M- $k \mathrm{TP}$ distributed. A transformation that applied to a mixed Poisson distribution always yields to a M- $k \mathrm{TP}$ distribution is defined.
\end{abstract}

\section{INTRODUCTION}

Count data often has larger variability than the one expected from the Poisson model. In Valero et al. (2010) it is pointed out that in many of those instances the data generating process can be modeled through a two stage process in which the distribution of each count would be Poisson but with an expected value that changes from count to count. By modeling the distribution of the Poisson expectation one is naturally lead to the use of Poisson mixture models.

These models have the advantatge that they provide a simple mechanistic explanation that lets one interpret the mixing distribution as the frequency distribution of a population characterizing the sample. That is useful for example when modelling word or species frequencies count data, in stylometry and in ecology, because the mixing distribution represents the word or species frequency distribution of the vocabulary or the population from which the sample was created (see, e.g. Sichel 1975, 1997).

2000 Mathematics Subject Classification. 60E05, 60E10, 60E07.

Key words and phrases. Count variable, Mixed distribution, Left-truncated distribution, Zero-modified distribution, Probability generating function. 
In applications one faces situations where one can not observe the counts smaller or equal than $k$, for $k \geq 0$, or where one needs to model the frequency of the values larger than $k$ apart. By resorting to the use of left-truncated mixed Poisson distributions, denoted here by $k \mathrm{~T}-\mathrm{MP}$, one is still allowed the mechanistic interpretation described above, which is lacking in the alternative models for this kind of data.

This paper characterizes by means of their probability generating functions (pgf's) the models that result from left-truncating at $k$ mixed Poisson distributions, denoted as $k \mathrm{~T}-\mathrm{MP}$, those that result from mixing Poisson distributions that have been previously left-truncated at $k$, denoted as M- $k \mathrm{TP}$, and those obtained by left-truncating at $k$ a mixture of Poisson distributions previously left-truncated at $i$, denoted as $k \mathrm{~T}-\mathrm{M}-i \mathrm{TP}$. The characterizations obtained allow on the one side to classify any given parametric model as belonging or not in any of these clases based on the properties of its pgf. On the other side, they allow one to define new models of the required type by means of their pgf's.

The characterization of the $k \mathrm{~T}-\mathrm{MP}$ distributions is specially useful because if one assumes that the mixing distribution has probability zero at zero, which is true in the continuous case, it is possible to recuperate features of the untruncated mixed Poisson model from the pgf of the $k \mathrm{~T}-\mathrm{MP}$ model. In particular, we present a way to recover the first $k+1$ probabilities of the untruncated model starting from the pgf of the the $k \mathrm{~T}-\mathrm{MP}$ model. This is specially rellevant for example in the analysis of word or species frequency count data though 0T-MP models because the estimation of the size of the vocabulary or population is directly related to the estimation of the probability of zero of the corresponding untruncated model and hence, of the proportion of words or species unobserved in the data.

Böhning and Kuhnert (2006) proves that if the mixing distribution has a finite support and the truncation is at zero, the two sets of distributions obtained by either first truncating and then mixing or the other way around a non negative integer distribution, are the same set of distributions. Valero et al. (2010) proved that when the mixing distribution has infinite support and the mixed untruncated distribution is Poisson, this result is no longer true. Here it is proved that when one left-truncates at any non-negative integer value $k$ other than zero, every $k \mathrm{~T}-\mathrm{MP}$ distribution is a M- $k \mathrm{TP}$ distribution but not the other way around.

The shifted version and the factorial size-biased version of order $k+1$ of a count distribution are two alternative mechanisms of obtaining count distributions taking only integer values larger than $k$. From the characterizations presented in this paper it follows that the factorial size-biased version of order $k+1$ and, under a certain condition, the shifted version of order $k+1$ of a Poisson mixture are neither a $k \mathrm{~T}-\mathrm{MP}$ distribution nor a M- $k \mathrm{TP}$ distribution. A transformation that applied to a mixed Poisson distribution always yields a M-kTP distribution is proposed.

The paper is organized as follows. In Section 2 the background definitions are given, a known characterization of Poisson mixture distributions is presented 
and two useful transformations of a random variable are defined. In Section 3 the pgf's of the $k \mathrm{~T}$-MP models are characterized and a method to recuperate the pgf of the initial untruncated mixed Poisson model from the pgf of the $k \mathrm{~T}-\mathrm{MP}$ model is proposed. Section 4 characterizes the pgf's of the M- $k \mathrm{TP}$ distributions. Section 5 remarks that the set of M- $k \mathrm{TP}$ distributions includes the set of $k \mathrm{~T}-\mathrm{MP}$ distributions. It also characterizes the set of $k \mathrm{~T}-\mathrm{M}-i \mathrm{TP}$ distributions and proves that this set is included in the set of $k \mathrm{~T}-\mathrm{M}-(i+1) \mathrm{TP}$ distributions. Section 6 presents consequences of the characterizations that relate to alternative ways to create count random variables supported on the integers larger than $k$. The results presented in this paper relie on and generalize some of the results for the zero-truncated case obtained in Valero et al. (2010). All the proofs are presented in the Appendix with the exception of the one of Proposition 4.

\section{Premilinary CONCEPts And Definitions}

The probability generating function of a non-negative integer (count) random variable (r.v.) $N$ such that $P(N=i)=p_{i}$ is defined as:

$$
h(s)=E\left[s^{N}\right]=\sum_{i=0}^{+\infty} p_{i} s^{i}
$$

for any $s$ in the domain of convergence, which includes $(-1,1)$. The pgf of a r.v. depends only on its probability distribution, and two r.v.'s have the same pgf if, and only if, they have the same probability distribution. Any pgf verifies that $h(0)=p_{0}$ and that $h(1)=1$. Denoting the $i$-th derivative of $h(s)$ by $h^{(i)}(s)$, it is easy to check that $p_{i}=h^{(i)}(0) / i$ ! and that if the $i$-th factorial moment of that r.v. exists, i. e. if $E[X ! /(X-i) !]<+\infty$, then it is equal to $h^{(i)}(1)$.

A function such that all its derivatives $f^{(i)}(s)$ exist in an interval $(a, b)$ is said to be absolutely monotone (strictly absolutely monotone) in $(a, b)$ if, and only if,

$$
f^{(i)}(s) \geq(>) 0, \forall s \in(a, b) \text { and } i=0,1,2, \cdots .
$$

A real function $f(s)$ is a pgf if, and only if, it is absolutely monotone in $(0,1)$ and $f(1)=1$ (see Feller, 1971).

Given a count random variable $N$ and a positive integer number $k$, the lefttruncation at $k$ of $N$, denoted by $N^{k-t r}$, is defined to be the r.v. with probabilities

$$
P\left(N^{k-t r}=i\right)=P(N=i \mid N>k)=\frac{P(N=i)}{1-\sum_{j=0}^{k} P(N=j)} \quad i=k+1, k+2, \cdots .
$$

It is easy to check that if one denotes the pgf of $N$ by $h_{N}(s)$, the pgf of $N^{k-t r}$ is

$$
h_{N^{k-t r}}(s)=\frac{h_{N}(s)-\sum_{j=0}^{k} P(N=j) s^{j}}{1-\sum_{j=0}^{k} P(N=j)}
$$

which means that 


$$
h_{N^{k-t r}}(s)+\frac{\sum_{j=0}^{k} P(N=j) s^{j}}{1-\sum_{j=0}^{k} P(N=j)}=\frac{h_{N}(s)}{1-\sum_{j=0}^{k} P(N=j)} .
$$

The pgf of a Poisson distribution with mean parameter $\lambda$ is equal to $\exp (\lambda(s-1))$, and hence by (2), the pgf of the left-truncation at $k$ of a Poisson distribution is:

$$
h_{P o(\lambda)^{k-t r}}(s)=\frac{e^{\lambda(s-1)}-e^{-\lambda} \sum_{j=0}^{k} \frac{\lambda^{j}}{j !} s^{j}}{1-e^{-\lambda} \sum_{j=0}^{k} \frac{\lambda^{j}}{j !}}=\frac{e^{\lambda s}-\sum_{j=0}^{k} \frac{\lambda^{j}}{j !} s^{j}}{e^{\lambda}-\sum_{j=0}^{k} \frac{\lambda^{j}}{j !}}=\frac{Q_{k}(s ; \lambda)}{Q_{k}(1 ; \lambda)},
$$

where

$$
Q_{k}(s ; \lambda)=e^{\lambda s}-\sum_{j=0}^{k} \frac{\lambda^{j}}{j !} s^{j} .
$$

A zero-modification of a r.v. $N$, denoted by $N^{*}$, is a r.v. with a distribution that is a mixture of the distribution of $N$ and the degenerated distribution at zero. Thus, one has that

$$
P\left(N^{*}=k\right)=\left\{\begin{array}{l}
\epsilon+(1-\epsilon) P(N=k) \text { if } k=0 \\
(1-\epsilon) P(N=k) \text { if } k \geq 1,
\end{array}\right.
$$

for a given value $\epsilon$ satisfying that $\epsilon+(1-\epsilon) P(N=0) \geq 0$. Positives, negatives, values of $\epsilon$ yield r.v.'s with probability at zero larger, smaller, than $N$ (see Johnson et al. 2005). The fact that the left-truncation at $k$ of a r.v. and the left-truncation at $k$ of any of its zero-modifications are equal will be used in Section 3.

This paper deals with mixed Poisson distributions, which are the result of assuming that the mean parameter of a Poisson distribution follows a given probability distribution. The following characterization is a reformulation of a result in Puri and Goldie (1979).

Proposition 1. A function $h_{N}(s)$ is the pgf of a r.v. $N$ with a mixed Poisson distribution different from the degenerated distribution at zero if, and only if, it is such that:

a) $h_{N}(1)=1$,

b) it is analytical in $(-\infty, 1)$,

c) all the coefficients of the series expansion of $h_{N}(s)$ about any point $s_{0}$ in $(-\infty, 1)$ are strictly positive, or what is the same, $h_{N}(s)$ is strictly absolutely monotone in $(-\infty, 1)$.

Remember that a given function is called analytical in the neighborhood of $x=a$ if an expansion of $f(x)$ as a convergent power series in $(x-a)$ is possible in this neighborhood (see Courant and John, 1965). It follows that if $N$ has a mixed Poisson distribution and $h_{N}(s)$ denotes its pgf, then $h_{N}(s)$ is a non-negative increasing function which limit is established in the following proposition. 
Lemma 1. If $N$ is a mixed Poisson distributed r.v. with pgf $h_{N}(s)$ then

$$
\lim _{s \rightarrow-\infty} h_{N}(s)=\epsilon,
$$

where $\epsilon$ is the probability that the mixing distribution takes the zero value.

This proposition is a consequence of the fact that if $U$ denotes the distribution function of the mixing distribution and it is assumed that $U(0)=\epsilon$, denoting by $U_{0}$ the distribution function of the zero-truncation of $U$ one has that:

$$
h_{N}(s)=\epsilon+(1-\epsilon) \int_{0}^{\infty} e^{\lambda(s-1)} d U_{0}(\lambda),
$$

which tends to $\epsilon$ when $s$ tends to $-\infty$.

It is important to observe that in the case where $\epsilon>0, N$ may be posed as a zero-modification of a mixed Poisson distribution with a pgf with limit zero at $-\infty$. For more details on the Poisson distribution and on distributions resulting from mixing and truncating it see Johnson et al. (2005).

The normalization of a given function $f(s)$ such that $f(1) \neq 0$ is defined to be $f(s) / f(1)$. When it exist, the derivative transformation of $f(s)$ is defined to be the normalization of the first derivative of $f(s)$, and the integral transformation of $f(s)$ is defined to be the normalization of the definite integral of $f(s)$ between zero and a positive value $s$. In what follows, starting from a r.v. $N$ new r.v.'s are defined through the derivative and integral transformation of its pgf, $h_{N}(s)$.

DEFINITION 1: The derivative transformation of a count r.v. $N$ with $E(N)<$ $+\infty$ and with pgf $h_{N}(s)$ is the r.v. $N^{\prime}$, with pgf:

$$
h_{N^{\prime}}(s)=\frac{h_{N}^{\prime}(s)}{h_{N}^{\prime}(1)} \text {. }
$$

Observe that if $N^{\prime}$ is the derivative transformation of $N$, then:

$$
h_{N^{\prime}}^{(j)}(s)=\frac{h_{N}^{(j+1)}(s)}{h_{N}^{\prime}(1)},
$$

and hence if $h_{N}^{(j)}(0)=0$ for $j=0,1, \cdot, k$, then $h_{N^{\prime}}^{(j)}(0)=0$ for $j=0,1, \cdots, k-1$. Thus, if $N$ is supported on the integers larger than or equal to $k$ with $k \geq 1$, then $N^{\prime}$ is supported on the integers larger than or equal to $k-1$; if $N$ is supported on the integers larger than or equal to zero then $N$ and $N^{\prime}$ have the same support. Moreover, if $h_{N}^{(j)}(1)<+\infty$, for $j=0,1, \cdots, k$, then $h_{N^{\prime}}^{(j)}(1)<+\infty$, for $j=0,1, \cdots, k-1$.

DEFINITION 2: The integral transformation of a count r.v. $N$ with pgf $h_{N}(s)$ is the r.v. $M$, with pgf:

$$
h_{M}(s)=\frac{\int_{0}^{s} h_{N}(t) d t}{\int_{0}^{1} h_{N}(t) d t} .
$$


Note that $\int_{0}^{1} h_{N}(t) d t=E(1 /(N+1))$, which for count r.v.'s is always finite and smaller than one.

If $M$ is an integral transformation, then $h_{M}(0)=0$ and thus $M$ has probability zero at zero. Moreover,

$$
h_{M}^{(j)}(s)=\frac{h_{N}^{(j-1)}(s)}{\int_{0}^{1} h_{N}(t) d t}, \quad \forall j \geq 1,
$$

from where one has that if $h_{N}^{(j)}(0)=0$, for $j=0,1, \cdots, k$, then $h_{M}^{(j)}(0)=0$, for $j=0,1, \cdots, k+1$. Thus, if $N$ is supported on integers larger than or equal to $k, k \geq 0$, then its integral transformation, $M$, is supported on integers larger than or equal to $k+1$. Moreover, if $h_{N}^{(j)}(1)<+\infty$, for $j=0,1, \cdots, k$, then $h_{M}^{(j)}(1)<+\infty$, for $j=0,1, \cdots, k+1$.

Intuitively it seems that the derivative and the integral transformations are inverse, in what follows it is argued that this is true under a given condition. It is easy to check that two r.v.'s have the same derivative transformation if, and only if, one is a zero-modification of the other one with a positive $\epsilon$. Thus, if one restricts atention to the set of pgf's such that $h(0)=0$ and $h^{\prime}(1)<+\infty$, the derivative transformation is injective. The integral transformation is defined in the set of pgf's of count distributions and, in that set, it is injective. Given that any pgf of a count distribution is the derivative transformation of a pgf of a count distribution with finite mean and probability zero at zero, one has that if one considers as initial space the set of pgf's of count distributions with finite mean and probability zero at zero and as a final space the set of pgf's of count distributions, both the derivative and the integral transformations defined are one the inverse of the other one.

Observe that if $h_{N}(s)$ is (strictly) absolutely monotone, the pgf of its derivative transformation is also (strictly) absolutely monotone. As a consequence, the derivative transformation of a mixed Poisson distribution is a mixed Poisson distribution. On the other hand, in Section 6 it will be found that the integral transformation of a mixed Poisson distribution is an strictly positive distribution, which is not necessarily a zero-truncated mixed Poisson distribution.

\section{Left-Truncation of a Mixed Poisson Distribution}

3.1. Characterization of KT-MP distributions. The next theorem characterizes the distributions obtained by left-truncating at $k$ a mixed Poisson distribution with the first $k+1$ moments finite.

Theorem 1. A function $h(s)$ is the pgf of a kT-MP distributed r.v. with the first $k+1$ moments finite if, and only if, it verifies that:

a) $h^{(i)}(0)=0$ for $i=0,1,2, \cdots, k$;

b) $h(1)=1$ and $h^{(i)}(1)<+\infty$, for $i=1,2, \cdots, k+1$;

c) it is analitical at $(-\infty, 1)$; 
d) all the coefficients of the series expansion of $h(s)$ about any point $s_{0}$ in $(-\infty, 1)$ are strictly positive except the first $k+1$ coefficients that may be negative, or what is the same, $h^{(k+1)}(s)$ is strictly absolutely monotone in $(-\infty, 1)$

e) there exists a polinomial function $q_{k}(s)=L_{0}+L_{1} s+\cdots+L_{k} s^{k}$ with $L_{i}>0$, for $i=0,1, \cdots, k$ such that

$$
\lim _{s \rightarrow-\infty}\left(h(s)+q_{k}(s)\right)=0 .
$$

Proposition 1 indicates that given any function $h(s)$ such that $h^{(k+1)}(1)<+\infty$ and that it satisfyies conditions $c$ ) and $d$ ) in Theorem 1 , the normalization of $h^{(k+1)}(s), h^{(k+1)}(s) / h^{(k+1)}(1)$, is the pgf of a non-degenerated mixed Poisson r.v. The next result is a direct consequence of Step 2 of the proof of Theorem 1.

Corollary 1. Let $N$ be a count r.v. with $p g f h_{N}(s)$ and let $N^{\prime}$ be its derivative transformation. Then, if $N$ has a $(k+1) T-M P$ distribution with finite first $k+2$ moments for a given $k \geq 1$, then $N^{\prime}$ has a $k T$-MP distribution with finite first $k+1$ moments.

Observe that by applying Corollary 1 recursively one has that if $N$ has a $(k+1) \mathrm{T}$-MP distribution with first $k+2$ moments finite, then the normalization of $h_{N}^{(j)}(s)$ for any $1 \leq j \leq k+1$ is the pgf of a $(k+1-j)$ T-MP distribution. With the understanding that a $(-1) \mathrm{TP}$ distribution is a MP distribution, it also follows that the normalization of $h_{N}^{k+2)}(s)$ is the pgf of a mixed Poisson distribution.

\subsection{Determination of the untruncated mixed Poisson model from a} $k$ T-MP. An important and interesting property of the distributions in the $k \mathrm{~T}$ MP family is that if the mixing distribution has probability zero at zero, one can recover the first $k+1$ probabilities of the untruncated mixed Poisson model. To determinate the untruncated mixed Poisson model is very useful. For example in the analysis of words or species frequency count data though 0T-MP models, computing the probability at zero of the corresponding untruncated mixed Poisson model allows one to determinate the proportion of unobserved words or species, and therefore the size of the vocabulary or of the population. This property is a consequence of the following theorem which is more general, since it refers to any kind of mixing distribution not only those with probability zero at zero.

Theorem 2. Let $h(s)$ be the pgf of the left-truncation at $k$ of a mixed Poisson r.v. $N$ with the first $k+1$ moments finite and with a pgf such that $\lim _{s \rightarrow-\infty} h_{N}(s)=$ $\epsilon \in[0,1)$. Starting from $h(s)$ it is possible to determine $h_{N}(s)$ and $P(N=j)$ for $j=0,1,2, \cdots, k$ through:

$$
P(N=0)=\epsilon+\frac{L_{0}(1-\epsilon)}{1+\sum_{i=0}^{k} L_{i}},
$$




$$
P(N=j)=\frac{L_{j}(1-\epsilon)}{1+\sum_{i=0}^{k} L_{i}}, \quad \text { for } \quad j=1,2, \cdots, k
$$

and

$$
h_{N}(s)=\epsilon+(1-\epsilon) \frac{h(s)+q_{k}(s)}{1+q_{k}(1)}
$$

where $q_{k}(s)$ is the polynomial of condition e) in Theorem 1; which coefficients $L_{j}$ can be calculated though:

$$
L_{k}=\frac{-1}{k !} \lim _{s \rightarrow-\infty} h^{(k)}(s)
$$

and

$$
L_{j}=\frac{-1}{j !} \lim _{s \rightarrow-\infty}\left(h(s)+\sum_{i=j+1}^{k} L_{i} s^{i}\right)^{(j)} \quad \text { for } \quad j=0,1, \cdots k-1 .
$$

Observe that from Lemma $1 \epsilon$ is equal to the probability at zero of the mixing distribution, and thus if it is equal to zero by (12) the pgf of the corresponding $k \mathrm{~T}$ MP distribution completely determines the pgf of the corresponding untruncated mixed Poisson distribution.

EXAMPLE 1: Let us consider the function:

$$
h(s)=\frac{s^{2}(8-3 s)}{5(s-2)^{2}},
$$

which is the pgf of a r.v. with probability zero at zero and at one because $h(0)=0$ and $h^{\prime}(0)=0$. Given that $h(s)$ satisfies the conditions of Theorem 1 , it is the pgf of the left-truncation at one of a mixed Poisson r.v. $N$ with pgf $h_{N}(s)$. From (13) and (14) one has that

$$
L_{1}=\frac{3}{5}, \quad \text { and that } \quad L_{0}=\frac{4}{5} .
$$

Moreover, if one assumes that $\lim _{s \rightarrow-\infty} h_{N}(s)=\epsilon$, by (12) one has that

$$
h_{N}(s)=\epsilon+(1-\epsilon) \frac{h(s)+\frac{4}{5}+\frac{3}{5} s}{1+\frac{7}{5}}=\epsilon+(1-\epsilon) \frac{(4-s)}{3(s-2)^{2}},
$$

and by $(10)$ and (11), $P(N=0)=\epsilon+(1-\epsilon) / 3$ and $P(N=1)=(1-\epsilon) / 4$. For the particular case where $\epsilon=0$, and taking into account (15), one has that $h(s)$ is the left-truncation at one of a mixture of two negative binomial distributions with $\alpha=2$ and $\theta=1 / 2$. 


\section{Mixture of Left-Truncated Poisson Distributions}

In order to prove the main result of this section, we need to establish first the following result.

Proposition 2. Let $N$ be a count r.v. with pgf $h_{N}(s)$ such that $h_{N}(0)=0$, and let $N^{\prime}$ be its derivative transformation. Given $k \geq 1, N$ is $M-k T P$ distributed with first $k+1$ moments finite if, and only if, $N^{\prime}$ is $M-(k-1) T P$ distributed with first $k$ moments finite.

The next theorem states that the first four conditions in Theorem 1 are enough to characterize the pgf's of M- $k$ TP distributions.

Theorem 3. A function $h(s)$ is the pgf of a $M-k T P$ distribution with the first $k+1$ moments finite if, and only if, it verifies that:

a) $h^{(i)}(0)=0$ for $i=0,1,2, \cdots, k$;

b) $h(1)=1$ and $h^{(i)}(1)<+\infty$ for $i=1,2, \cdots, k+1$;

c) it is analitical at $(-\infty, 1)$;

d) all the coefficients of the series expansion of $h(s)$ about any point $s_{0}$ in $(-\infty, 1)$ are strictly positive exept the first $k+1$ coefficients that may be negative, or what is the same, $h^{(k+1)}(s)$ is strictly absolutely monotone in $(-\infty, 1)$.

The next consequence of Theorem 3 helps recognize pgf's of M- $k$ TP distributions.

Corollary 2. Let $k$ be a positive integer value. A function $h(s)$ such that $h^{(i)}(0)=$ 0 for $i=0,1, \cdots, k$ and such that $h^{(i)}(1)<+\infty$ for $i=1,2 \cdots, k+1$ is the pgf of a M-kTP distribution with first $k+1$ moments finite if, and only if, the normalization of $h^{(k+1)}(s)$ is the pgf of a mixed Poisson distribution.

\section{Consequences of the Characterizations}

5.1. The set $k \mathbf{T}-\mathbf{M P}$ is a subset of the set $\mathbf{M}-k \mathbf{T P}$. From Theorems 1 and 3 it follows that the set of $k \mathrm{~T}-\mathrm{MP}$ distributions is strictly included in the set of M- $k$ TP distributions.

\section{Corollary 3.}

a) Every $k T-M P$ distribution is a $M-k T P$ distribution.

b) A M-kTP distribution with pgf $h(s)$ is a $k T-M P$ distribution if, and only if, there exists a polynomial of degree $k$ with positive coefficients $q_{k}(s)$ such that $h(s)+q_{k}(s)$ has limit zero at $-\infty$.

EXAMPLE 2: Consider the negative binomial distribution, with pgf:

$$
h(s)=\left(\frac{1-\theta}{1-\theta s}\right)^{\alpha}, \quad \text { where } 0<\theta<1, \alpha>0,
$$


the logarithmic series distribution, with pgf:

$$
h(s)=\frac{\ln (1-\theta s)}{\ln (1-\theta)}, \quad \text { where } \quad 0<\theta<1,
$$

and the Hermite distribution, with pgf:

$$
h(s)=e^{\alpha(s-1)+\beta\left(s^{2}-1\right)}, \quad \text { where } \quad \alpha, \beta>0 .
$$

The negative binomial and the Hermite distributions are supported on the nonnegative integers and the logarithmic series distribution is supported on the positive integers. Thus, the zero-truncation of the logarithmic series distribution is equal to the untruncated distribution. The top pannel of Figure 1 presents these pgf functions for a particular set of values for $\theta, \alpha$ and $\beta$, while the central and bottom pannels present the pgf's of their left-truncations at zero and one.

Given that the negative binomial distribution is a mixed Poisson distribution, its zero-truncation and its left-truncation at one are particular cases of $k \mathrm{~T}-\mathrm{MP}$ distributions and thus they are also M- $k \mathrm{TP}$ distributions.

Looking at the pgf of the Hermite distribution one concludes that it can not be a mixed Poisson distribution because the first derivative of its pgf takes the zero value at a point $s_{0} \in(-\infty, 1)$. Its zero-truncation is neither the zero-truncation of a Poisson mixture nor a mixture of zero-truncated Poisson distributions, because its first derivative also takes the value zero at some point in $(-\infty, 1)$ and thus condition $d$ ) of Theorems 1 and 3 is not verified. With respect to its left-truncation at one, it is not the left-truncation at one of a mixed Poisson distribution because condition $e$ ) of Theorem 1 is not satisfied, and even though it is not possible to apreciate it in Figure 1, it can be checked that it is not a mixture of Poisson distributions left-truncated at one either.

The logarithmic series distribution is not a Poisson mixture because its pgf takes negative values, it is not the zero-truncation of a Poisson mixture because its pgf does not have a finite limit at $-\infty$, but it can be checked that it is a mixture of zero-truncated Poisson distributions. Given that the left-truncation at one of the logarithmic series distribution satisfies the conditions of Theorem 3, it is a mixture of Poisson distributions left-truncated at one, but it is not the lefttruncation at one of a mixed Poisson distribution because it does not satisfy condition $e$ ) in Theorem 1 with $k=1$. Nevertheless, by construction it is the left-truncation at one of a mixture of zero-truncated Poisson distributions. The results obtained for these three particular models are summarized in Table 1.

5.2. Repeated combination of mixing and truncation. To truncate at $k$ a mixture of Poisson distributions previously left-truncated at $i$ with $i \geq k$ has no efect, leaving one with a distribution in M-iTP. In this subsection the distributions obtained by left-truncating at $k$ a mixture of Poisson distributions previously left-truncated at $i$ for $0<i<k$, denoted by $k \mathrm{~T}-\mathrm{M}-i \mathrm{TP}$, are characterized. 

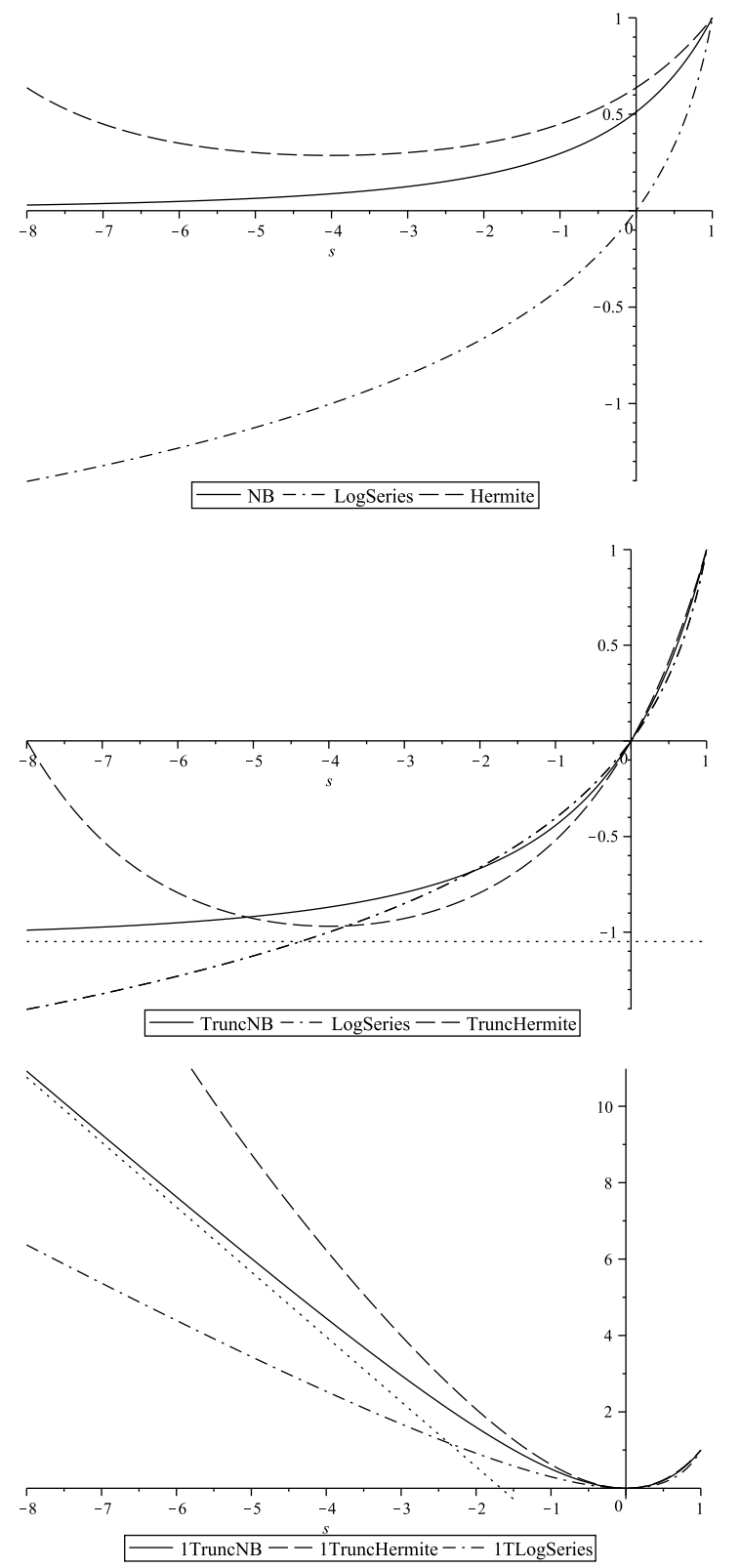

Figure 1. The top pannel presents the pgf's of the negative binomial with $(\theta=0.2, \alpha=3)$, of the logarithmic series with $\theta=0.75$, and of the Hermite distribution with $(\alpha=0.5, \beta=1 / 20)$. The central and bottom pannels present the pgf's of the corresponding left-truncations at zero and one. 
JORDI VALERO, MARTA PÉREZ-CASANY AND JOSEP GINEBRA

\begin{tabular}{|c|c|c|}
\hline Distribution & Degree of truncation & Type \\
\hline N. B. & untruncated & MP \\
& $\begin{array}{c}\text { zero-truncated } \\
\text { left-truncated at one }\end{array}$ & 0T-MP and M-0TP \\
& 1T-MP and M-1TP \\
\hline Hermite & zero-truncated & not MP \\
& neither 0T-MP nor M-0TP \\
& left-truncated at one & neither 1T-MP nor M-1TP \\
\hline log. series & untruncated & M-0TP but not 0T-MP \\
& left-truncated at one & M-1TP but not 1T-MP \\
\hline
\end{tabular}

TABLE 1. Summary of Example 2. Note that the logarithmic series distribution and its zero-truncation are the same distribution.

The next Proposition looks into the derivative transformation of a $k \mathrm{~T}-\mathrm{M}-i \mathrm{TP}$ distribution with $i<k$. Previously, the following lemma is required.

Lemma 2. If $N$ is a count r.v. with $p g f h_{N}(s)$, the derivative transformation of the left-truncation at $k$ of $N$ has the same probability distribution as the lefttruncation at $k-1$ of the derivative transformation of $N, N^{\prime}$.

Proposition 3. A count r.v. $N$ is kT-M-iTP distributed if, and only if, its derivative transformation is $(k-1) T-M-(i-1) T P$ distributed.

Appling Proposition 3 consecutively $(i+1)$ times, one obtains the following characterization of the $k \mathrm{~T}-\mathrm{M}-i \mathrm{TP}$ distributions. Note that to normalize the derivative of a given function consecutively $i+1$ times is equivalent to normalizing its $(i+1)$-th derivative.

Corollary 4. A count r.v. $N$ with $p g f h_{N}(s)$ is kT-M-iTP distributed if, and only if, the normalization of $h_{N}^{(i+1)}(s)$ is the pgf of a $(k-i-1) T$-MP distributed r.v.

The following theorem is the main result of this section. It proves that between the sets $k \mathrm{~T}-\mathrm{MP}$ and M- $k \mathrm{TP}$ one can consider $k$ intermediate sets obtained through repeated combinations of mixing and truncation.

Theorem 4. For any given positive integer value $k \geq 0$, one has that:

$$
\begin{aligned}
k T-M P \subset k T-M-(0 T P) \subset k T-M-(1 T P) \subset \cdots & \subset k T-M-((k-1) T P) \\
& \subset k T-M-(k T P)=M-k T P .
\end{aligned}
$$


EXAMPLE 2 (cont): Given that the left-truncation at one of the negative binomial distribution is a 1T-MP distribution, it is also a 1T-M-0TP distribution and a M-1TP distribution. The left-truncation at one of the logarithmic series distribution is not a 1T-MP distribution as already said, but by definition it is a 1T-M-0TP distribution and thus it is a M-1TP distribution. The Hermite distribution is not a M-1TP distribution, and hence it is neither a 1T-MP distribution nor a 1T-M-0TP distribution.

\section{On ALternative WAYS TO CREATE R.V.'S SUPPORTED ON THE INTEGERS LARGER THAN $k$}

There are ways different from left-truncating at $k$ to transform an initial count r.v. into a r.v. supported on the non-negative integers strictly larger than $k$. In this section we consider the shift version of order $k+1$ and the factorial size-biased version of a r.v., because these are the more natural ones. Given that these two transformations do not yield in general to r.v's which are M- $k$ TP distributed, and alternative transformation is first proposed.

6.1. On r.v.'s obtained by integral transformation. Proposition 2 states that a r.v. $N$ is M- $k$ TP distributed if, and only if, its derivative transformation is $\mathrm{M}-(k-1) \mathrm{TP}$ distributed. The next result is the analogous one for the integral transformations.

Proposition 4. If $M$ is the integral transformation of a given count r.v. $N$, then

a) $M$ is $M-0 T P$ distributed if, and only if, $N$ is mixed Poisson distributed with finite mean,

b) $M$ is $M-(k+1) T P$ distributed if, and only if, $N$ is $M-k T P$ distributed.

The first part of the proposition is proved in Proposition 3 of Valero et al. (2010). To see that $b$ ) holds, observe that from (9) one has that $h_{N}(s)$ verifies the conditions of Theorem 3 for $k=k_{0}$ if, and only if, $h_{M}(s)$ verifies the same conditions for $k=k_{0}+1$.

If $N$ is M- $k$ TP distributed, Proposition 4 ensures that its integral transformation, $M$, is M- $(k+1) \mathrm{TP}$ distributed, but it can also be $(k+1) \mathrm{T}-\mathrm{M}-k \mathrm{TP}$ distributed, which happens only if $N$ is $k \mathrm{~T}-\mathrm{M}-(k-1) \mathrm{TP}$ distributed. As a consequence of Proposition 4 , by applying the integral transformation to a M- $(k-1) \mathrm{TP}$ distribution one always obtains a M- $k \mathrm{TP}$ distribution. Instead, the transformations considered in Subsections 6.2 and 6.3 will not always lead to M- $k$ TP distributions.

Next we present an example of a distribution that is in M-1TP but neither in 1T-MP nor in 1T-M-0TP.

EXAMPLE 3: Let us define the integral logarithmic series distribution to be the integral transformation of the logarithmic series distribution, which is supported on the integer values larger than or equal to two and has a pgf: 


$$
h(s)=\left(s-\frac{1}{\theta}\right) \ln (1-\theta s)-s, \text { where } 0<\theta<1 .
$$

Given that the logarithmic series distribution is not the zero-truncation of a mixed Poisson distribution, by Corollary 1 one has that the integral logarithmic series distribution is not the left-truncation at one of a mixed Poisson distribution, 1TMP, and by Lemma 2, it is neither a 1T-M-0TP distribution. Nevertheless, it can be checked that the integral logarithmic series distribution is a mixture of Poisson distributions left-truncated at one, M-1TP, because its derivative transformation, the logarithmic series distribution, is a mixture of zero-truncated Poisson distributions. This can be appreciated in Figure 2, presenting the derivative transformation of the left-truncation at one of the integral logarithmic series distributions with $\theta=0.85$.

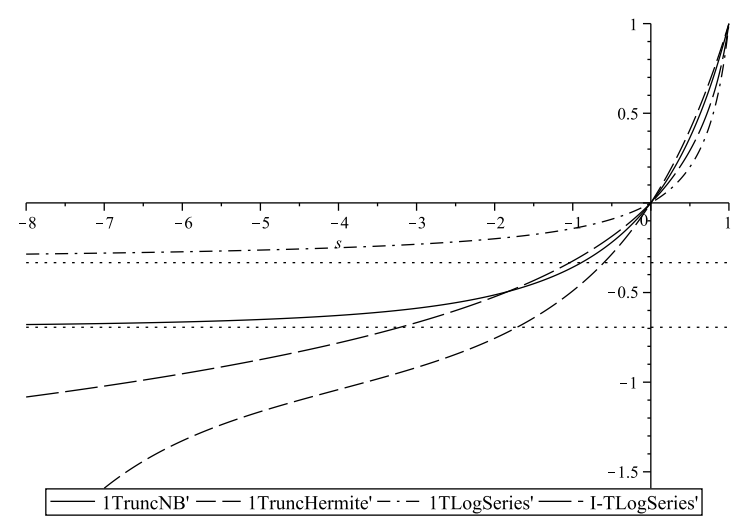

FiguRE 2. Derivative transformation of the lefttruncation at one of the pgf of the integral logaritmic series with $\theta=0.85$.

6.2. The Shifted version of order $k+1$. The shifted version of order $k+1$ of a count r.v. $N$, with $\operatorname{pgf} h_{N}(s)$, is defined to be the r.v. $M$ such that

$$
P(M=i)=P(N=i-(k+1)), \quad \text { for } \quad i=k+1, k+2, \cdots,
$$

and hence with pgf:

$$
h_{M}(s)=s^{k+1} h_{N}(s) .
$$

Proposition 5. Let $M$ be the shifted version of order $k+1$ of a Poisson mixture $N$ with $\operatorname{pgf} h_{N}(s)$, and let $\epsilon$ be the limit when $s$ tends to $-\infty$ of $h_{N}(s)$. If condition

$$
\lim _{s \rightarrow-\infty} s^{k+1}\left(h_{N}(s)-\epsilon\right)=0
$$

holds, then $M$ is neither $k T-M P$ distributed nor $M-k T P$ distributed. 
EXAMPLE 4: The Tweedie-Poisson model, first considered by Hougaard (1997), is a three parameter Poisson mixture model that arises by assuming that the mean of a Poisson distribution follows a three parameter Tweedie distribution with non-negative support. Its pgf is:

$$
h(s)=e^{\frac{\alpha(1-\beta)}{\beta}\left((1-\alpha)^{\beta}-(1-\theta s)^{\beta}\right)},
$$

where $\beta \in(-\infty, 1), \alpha \in(0,+\infty)$ and $\theta \in(0,1)$.

- For $\beta \in(0,1), h(s)$ has limit zero at $-\infty$ and condition (16) is verified for any $k \geq 0$. Consequently, the shifted version of any order of a TweediePoisson distribution with $\beta \in(0,1)$ is neither a $k \mathrm{~T}-\mathrm{MP}$ nor a M- $k \mathrm{TP}$. In particular this holds for the inverse-Gaussian Poisson model, obtained when $\beta=1 / 2$.

- The limit of (17) when $\beta$ tends to zero is the pgf of a negative binomial distribution in (15). In that case, the limit of $h(s)$ at $-\infty$ is equal to zero. If $k<\alpha-1$ condition (16) is satisfied and the corresponding shifted version of the negative binomial distribution is neither $k \mathrm{~T}-\mathrm{MP}$ distributed nor M- $k$ TP distributed.

- For $\beta \in(-\infty, 0)$ one obtains the Pólya-Aeppli models for which the limit of $(17)$ at $-\infty$ is equal to $\exp \left\{\alpha(1-\beta)(1-\alpha)^{\beta} / \beta\right\}$. If $\beta \leq-3$, condition (16) is satisfied when $k<-\beta-1$ and in that case, the corresponding shifted version of the Pólya-Aeppli is neither in $k \mathrm{~T}-\mathrm{MP}$ nor in M- $k \mathrm{TP}$.

In Valero et al. (2010) it is proved that the parameter space of the zero-truncated Tweedie-Poisson model can be made larger than the one for the Tweedie-Poisson mixture model. It is also proved that the distributions forming the extended part of the model are not 0T-MP and that other than when $\beta=0$, they are not M-0TP either.

6.3. The factorial size-biased version of order $k+1$. Given a count random variable $N$ with the first $k+1$ moments finite and with $\operatorname{pgf} h_{N}(s)$, the factorial size biased version of order $k+1$ of $N$ is defined to be its weighted version with weight:

$$
w(i)=i(i-1) \cdots(i-k), \quad \text { for } \quad i=0,1, \cdots .
$$

Hence, it is the r.v. $M$ with probability mass function:

$$
P(M=i)=\frac{i(i-1)(i-2) \cdots(i-k)}{h_{N}^{(k+1)}(1)} P(N=i),
$$

and therefore with $P(M=i)=0$ for $i=0,1,2 \cdots$, and with pgf:

$$
h_{M}(s)=\frac{s^{k+1} h_{N}^{(k+1)}(s)}{h_{N}^{(k+1)}(1)} .
$$


Proposition 6. If $M$ is the factorial size-biased version of order $k+1$ of a Poisson mixture $N$ with the first $k+1$ moments finite, then $M$ is neither $k T-M P$ distributed nor $M-k T P$ distributed.

\section{Conclusions}

In this work three types of probability models have been characterized. The one obtained by left-truncating at $k$ a mixed Poisson distribution, $k \mathrm{~T}-\mathrm{MP}$, the one obtained by mixing left-truncated Poisson distributions, M- $k \mathrm{TP}$, and the one obtained by left-truncating mixtures of previously left-truncated Poisson distributions, $k \mathrm{~T}-\mathrm{M}-i \mathrm{TP}$. To be able to recognize the $k \mathrm{~T}-\mathrm{MP}$ model is of special interest if the mixing distribution is continuous, because for this type of models one is allowed to recover the first probabilities of the untruncated model and therefore some features associated with the mixing distribution.

From the characterizations obtained one has that the set $k \mathrm{~T}-\mathrm{MP}$ is strictly included in the set M- $k$ TP. It also turns out that between these two sets one can consider $k$ intermediate sets obtained through repeated combinations of mixing and truncating. Moreover, it is proved that the factorial size-biased version of order $k+1$ and under a certain condition, the shifted version of order $k+1$ of a count r.v. are neither $k \mathrm{~T}-\mathrm{MP}$ distributed nor M- $k \mathrm{TP}$ distributed. A transformation that applied to a mixed Poisson distribution always yields to a M- $k \mathrm{TP}$ distribution is proposed.

It might be of interest to extend the results obtained in this paper for distributions that are mixtures of models for non negative integer data partically closed under addition (see, Puig and Valero, 2006, JASA), because their pgf's have an exponential form and the mixing parameters only appear in the exponent as a multiple of a function of $s$, very much alike what one has for Poisson mixtures.

\section{ApPendix: ProOfS}

Proof of Theorem 1: If $h(s)$ is the pgf of a r.v. with the first $k+1$ moments finite and with a $k \mathrm{~T}-\mathrm{MP}$ distribution, the first four conditions of the theorem hold as a straight consequence of (2) and of Proposition 1. To see that condition e) holds observe that by (3) one has that

$$
h(s)+\frac{\sum_{j=0}^{k} P(M=j) s^{j}}{1-\sum_{j=0}^{k} P(M=j)}=\frac{h_{M}(s)}{1-\sum_{j=0}^{k} P(M=j)},
$$

where $h_{M}(s)$ is the pgf of the corresponding untruncated Poisson mixture. Thus, if $h_{M}(s)$ has limit zero at $-\infty$, then $h(s)$ satisfies condition $\left.e\right)$ with $q_{k}(s)=$ $L_{0}+L_{1} s+L_{2} s^{2}+\cdots+L_{k} s^{k}$, where

$$
L_{i}=\frac{P(M=i)}{1-\sum_{j=0}^{k} P(M=j)}, \quad \text { for } \quad i=0,1,2, \cdots k .
$$


If otherwise the limit of $h_{M}(s)$ at $-\infty$ is equal to a strictly positive real number $\epsilon$, then $h(s)$ satisfies condition $e)$ with $q_{k}(s)$ being the polinomial with $L_{i}$ as in (21) for all $i \geq 1$ and with constant term:

$$
L_{0}=\frac{P(M=0)-\epsilon}{1-\sum_{j=0}^{k} P(M=j)} .
$$

The reverse implication, that a function verifing the five conditions of the theorem is always the pgf of a $k \mathrm{~T}-\mathrm{MP}$ r.v. with the first $k+1$ moments finite, is proved by induction. When $k=0$ the result follows from Theorem 1 of Valero et al. (2010). Assume that the result is true for $k=k_{0}$, and assume that $h(s)$ verifies the five conditions of the theorem for $k=k_{0}+1$. To prove that in that case $h(s)$ is the pgf of a $\left(k_{0}+1\right)$-truncated mixed Poisson distribution the next four steps are followed:

1) A r.v. $N$ is defined through the derivative transformation of $h(s)$.

2 ) It is proved that $N$ is the left-truncation at $k_{0}$ of a mixed Poisson r.v. $M$, and $h_{M}(s)$ is obtained.

3) A zero-truncated mixed Poisson r.v. $M^{*}$ is defined by means of the integral transformation of $M$.

4) It is checked that the pgf of the left-truncation at $k_{0}+1$ of $M^{*}$ is equal to $h(s)$.

Step 1 By $a), c)$ and $d$ ) one has that

$$
h(s)=\sum_{j=1}^{+\infty} a_{k_{0}+1+j} s^{k_{0}+1+j},
$$

where $a_{k_{0}+1+j} \geq 0 j=1,2, \cdots$, and $\sum_{j=1}^{+\infty} a_{k_{0}+1+j}=1$. By $\left.e\right)$, there exists a polynomial

$$
q_{k_{0}+1}(s)=L_{0}+L_{1} s+\cdots+L_{k_{0}+1} s^{k_{0}+1}
$$

such that

$$
\lim _{s \rightarrow-\infty}\left(h(s)+q_{k_{0}+1}(s)\right)=0 .
$$

Let us denote by $N$ the r.v. with pgf:

$$
h_{N}(s)=\frac{h^{\prime}(s)}{h^{\prime}(1)} .
$$

Step 2 It is straightforward to check that $h_{N}(s)$ verifies conditions $\left.\left.a\right), b\right), c$ ) and $d$ ) of the theorem for $k=k_{0}$. To see that it also verifies condition $e$ ), consider the polynomial

$$
q_{k_{0}}(s)=\frac{q_{k_{0}+1}^{\prime}(s)}{h^{\prime}(1)}=\frac{L_{1}+2 L_{2} s+\cdots+\left(k_{0}+1\right) L_{k_{0}+1} s^{k_{0}}}{h^{\prime}(1)}
$$


and observe that

$\lim _{s \rightarrow-\infty}\left(h_{N}(s)+q_{k_{0}}(s)\right)=\frac{\lim _{s \rightarrow-\infty}\left(h^{\prime}(s)+q_{k_{0}+1}^{\prime}(s)\right)}{h^{\prime}(1)}=\frac{\lim _{s \rightarrow-\infty}\left(h(s)+q_{k_{0}+1}(s)\right)^{\prime}}{h^{\prime}(1)}$ and thus, condition $e$ ) is equivalent to:

$$
\lim _{s \rightarrow-\infty}\left(h(s)+q_{k_{0}+1}(s)\right)^{\prime}=0 .
$$

Given that $h(s)+q_{k_{0}+1}(s)$ has no more than $k_{0}+1$ critical points, there exists a value $s_{0} \in(-\infty, 1)$ such that $h(s)+q_{k_{0}+1}(s)$ is monotone in $\left(-\infty, s_{0}\right)$; to be consistent with $(25)$, the only possibility is that $\left(h(s)+q_{k_{0}+1}(s)\right)^{\prime}$ tends to zero at $-\infty$.

As a consequence, $N$ is the left-truncation at $k_{0}$ of a mixed Poisson r.v. $M$ with the first $k_{0}+1$ moments finite. Picking the mixed Poisson r.v. $M$ with pgf that has limit zero at $-\infty$, by (2) one has that

$$
h_{N}(s)=\frac{h_{M}(s)}{1-\sum_{j=0}^{k_{0}} P(M=j)}-\sum_{j=0}^{k_{0}} \frac{P(M=j)}{1-\sum_{j=0}^{k_{0}} P(M=j)} s^{j},
$$

and given that $h_{N}(s)$ verifies condition $\left.e\right)$ for $k=k_{0}$ with $q_{k_{0}}(s)$, by (27) the following equalities hold:

$$
\frac{(j+1) L_{j+1}}{h^{\prime}(1)}=\frac{P(M=j)}{1-\sum_{j=0}^{k_{0}} P(M=j)}, \text { for } j=0,1, \cdots k_{0} .
$$

Consequently,

$$
q_{k_{0}}(s)=\sum_{j=0}^{k_{0}} \frac{P(M=j)}{1-\sum_{j=0}^{k_{0}} P(M=j)} s^{j}, \quad \text { and } \quad 1+q_{k_{0}}(s)=\frac{1}{1-\sum_{j=0}^{k_{0}} P(M=j)} .
$$

Taking into account the last two equalities, (28) may also be expressed as:

$$
h_{N}(s)=\left(1+q_{k_{0}}(1)\right) h_{M}(s)-q_{k_{0}}(s),
$$

and thus,

$$
h_{M}(s)=\frac{h_{N}(s)+q_{k_{0}}(s)}{1+q_{k_{0}}(1)} .
$$

Step 3 Let $M^{*}$ be the integral transformation of $M$ with pgf:

$$
h_{M^{*}}(s)=\frac{\int_{0}^{s} h_{M}(t) d t}{\int_{0}^{1} h_{M}(t) d t} .
$$

As has been pointed out in Section 2, by definition $P\left(M^{*}=0\right)=h_{M^{*}}(0)=0$ and $M^{*}$ has the first $k_{0}+2$ moments finite. Taking into consideration (26), (27) and (29), one has that

$$
h_{M^{*}}(s)=\frac{\int_{0}^{s}\left(h^{\prime}(t)+q_{k_{0}+1}^{\prime}(t)\right) d t}{\int_{0}^{1}\left(h^{\prime}(t)+q_{k_{0}+1}^{\prime}(t)\right) d t}
$$


and thus that,

$$
h_{M^{*}}(s)=\frac{h(s)+q_{k_{0}+1}(s)-h(0)-q_{K_{0}+1}(0)}{h(1)+q_{K_{0}+1}(1)-h(0)-q_{k_{0}+1}(0)} .
$$

Given that $h(0)=0$ by condition $a$ ) and that by $(24) q_{k_{0}+1}(0)=L_{0}$, one has that

$$
h_{M^{*}}(s)=\frac{h(s)+q_{k_{0}+1}(s)-L_{0}}{1+q_{k_{0}+1}(1)-L_{0}}
$$

and thus that,

$$
h_{M^{*}}^{(j)}(s)=\frac{h^{(j)}(s)+q_{k_{0}+1}^{(j)}(s)}{1+q_{k_{0}+1}(1)-L_{0}}, \quad \forall j \geq 1 .
$$

From (32), and taking into account the relationship between the derivatives of a pgf evaluated at zero and the probabilities of the corresponding probability distribution, one has that

$$
P\left(M^{*}=j\right)=\frac{h_{M^{*}}^{(j)}(0)}{j !}=\frac{h^{(j)}(0)+q_{k_{0}+1}^{(j)}(0)}{j !\left(1+q_{k_{0}+1}(1)-L_{0}\right)}, \quad \forall j \geq 1 .
$$

Because of Proposition 3 of Valero et al. (2010), to check that $M^{*}$ is the zerotruncation of a mixed Poisson distribution it is enough to check that $h_{M^{*}}(s)$ has a finite limit at $-\infty$. But by (31) and (25) one has that

$$
\lim _{s \rightarrow-\infty} h_{M^{*}}(s)=\frac{-L_{0}}{1+q_{k_{0}+1}(1)-L_{0}}<+\infty .
$$

Step 4 By (2), (31) and (33), the pgf of the left-truncation at $k_{0}+1$ of $M^{*}$ is equal to:

$$
\begin{aligned}
h_{M^{*}}^{\left(k_{0}+1\right)-t r}(s) & =\frac{h_{M^{*}}(s)-\sum_{j=0}^{k_{0}+1} P\left(M^{*}=j\right) s^{j}}{1-\sum_{j=0}^{k_{0}+1} P\left(M^{*}=j\right)} \\
& =\frac{h(s)+q_{k_{0}+1}(s)-L_{0}-\sum_{j=1}^{k_{0}+1} \frac{h^{(j)}(0)+q_{k_{0}+1}^{(j)}(0)}{j !} s^{j}}{1+q_{k_{0}+1}(1)-L_{0}-\sum_{j=1}^{k_{0}+1} \frac{h^{(j)}(0)+q_{k_{0}+1}^{(j)}(0)}{j !}},
\end{aligned}
$$

and given that by condition $a$ ) one has that $h^{(j)}(0)=0$ for $j=0, \cdots, k_{0}+1$, and that by $(24)$ one has that $q_{k_{0}+1}^{(j)}(0) / j !=L_{j}$ for $j=1, \cdots, k_{0}+1$, it follows that

$$
h_{M^{*}}^{\left(k_{0}+1\right)-t r}(s)=h(s) .
$$

Proof of the Theorem 2: Let $h(s)$ be the pgf of the left-truncation at $k$ of a mixed Poisson r.v. $N$ with pgf $h_{N}(s)$ and such that $\lim _{s \rightarrow-\infty} h_{N}(s)=\epsilon \in[0,1)$. 
If $q_{k}(s)$ is the polinomial of condition $e$ ) of Theorem 1 , by (2) one has that:

$$
\begin{aligned}
0 & =\lim _{s \rightarrow-\infty}\left(h(s)+q_{k}(s)\right)=\lim _{s \rightarrow-\infty}\left(\frac{h_{N}(s)-\sum_{j=0}^{k} P(N=j) s^{j}}{1-\sum_{j=0}^{k} P(N=j)}+q_{k}(s)\right) \\
& =\frac{\epsilon(N=0)}{1-\sum_{j=0}^{k} P(N=j)}-\frac{P(N)}{1-\sum_{j=0}^{k} P(N=j)}+L_{0} \\
& +\lim _{s \rightarrow-\infty} \sum_{j=1}^{k}\left(\frac{-P(N=j)}{1-\sum_{j=0}^{k} P(N=j)}+L_{j}\right) s^{j} .
\end{aligned}
$$

This equation is true only if the following system of equations is satified:

$$
\left.\begin{array}{l}
\frac{\epsilon}{1-\sum_{j=0}^{k} P(N=j)}-\frac{P(N=0)}{1-\sum_{j=0}^{k} P(N=j)}=-L_{0} \\
\frac{-P(N=j)}{1-\sum_{j=0}^{k} P(N=j)}+L_{j}=0, \text { for } j=1,2, \cdots, k
\end{array}\right\}
$$

Adding the last $k$ equations and isolating the sum of the probabilities one has that

$$
\sum_{j=1}^{k} P(N=j)=\frac{(1-P(N=0)) \sum_{j=1}^{k} L_{j}}{1+\sum_{j=1}^{k} L_{j}}
$$

which gives that

$$
1-\sum_{j=0}^{k} P(N=j)=\frac{1-P(N=0)}{1+\sum_{j=1}^{k} L_{j}} .
$$

Substituing (34) in the first equation one has that:

$$
P(N=0)=\frac{\epsilon\left(1+\sum_{j=1}^{k} L_{j}\right)+L_{0}}{1+\sum_{i=0}^{k} L_{i}}=\epsilon+\frac{L_{0}(1-\epsilon)}{1+\sum_{i=0}^{k} L_{i}} .
$$

By substituing $P(N=0)$ in (34), after some calculus one has that:

$$
1-\sum_{j=0}^{k} P(N=j)=\frac{1-\epsilon}{1+\sum_{j=0}^{k} L_{j}}
$$

and thus that:

$$
P(N=j)=\frac{L_{j}(1-\epsilon)}{1+\sum_{i=0}^{k} L_{i}} \quad \text { for } \quad k=1,2, \cdots, k .
$$


To see that (12) is verified, observe that by (2), (36), (35) and (37) one has that

$$
\begin{aligned}
h_{N}(s) & =h(s)\left(1-\sum_{j=0}^{k} P(N=j)\right)+\sum_{j=0}^{k} P(N=j) s^{j} \\
& =\frac{h(s)(1-\epsilon)}{1+q_{k}(1)}+\epsilon+\frac{L_{0}(1-\epsilon)}{1+q_{k}(1)}+\sum_{j=1}^{k} \frac{L_{j}(1-\epsilon)}{1+q_{k}(1)} s^{j} \\
& =\epsilon+(1-\epsilon) \frac{h(s)+q_{k}(s)}{1+q_{k}(1)} .
\end{aligned}
$$

To calculate the coefficients of polinomial $q_{k}(s)$ starting from $h(s)$, let $M_{0}$ be a r.v. with distribution the untruncated mixed Poisson model associated to $h(s)$ with $\epsilon=0$. Thus, $h_{M_{0}}(s)$ is obtained from (12) with $\epsilon=0$ and given that it has to be constant and equal to zero in $(-\infty, 0]$, differentiating it one has that for all $i \geq 0$ :

$$
0=\lim _{s \rightarrow-\infty} h_{M_{0}}^{(i)}(s)=\lim _{s \rightarrow-\infty} \frac{h^{(i)}(s)+i ! L_{i}+\sum_{j=i+1}^{k} \frac{j !}{(j-i) !} L_{j} s^{j-i}}{1+q_{k}(1)},
$$

which implies that:

$$
L_{k}=\frac{-1}{k !} \lim _{s \rightarrow-\infty} h^{(k)}(s),
$$

and that for all $0 \leq i \leq k-1$,

$L_{i}=-\frac{1}{i !} \lim _{s \rightarrow-\infty}\left(h^{(i)}(s)+\sum_{j=i+1}^{k} \frac{j !}{(j-i) !} L_{j} s^{j-i}\right)=-\frac{1}{i !} \lim _{s \rightarrow-\infty}\left(h(s)-\sum_{j=i+1}^{k} L_{j} s^{j}\right)^{(i)}$.

Proof of Proposition 2: If $N$ has a M- $k$ TP distribution with the first $k+1$ moments finite, there exists a constant $t$ and a non-negative r.v. $\Gamma$ with mean equal to one and with probability density function $f(x)$ such that $\lambda=t \Gamma$ and

$$
h_{N}(s)=\int_{0}^{+\infty} \frac{Q_{k}(s ; t x)}{Q_{k}(1 ; t x)} f(x) d x
$$

Differentiating both sides one has that

$$
h_{N}^{\prime}(s)=\int_{0}^{+\infty} \frac{Q_{k-1}(s ; t x)}{Q_{k}(1 ; t x)} t x f(x) d x
$$

and in particular that:

$$
h_{N}^{\prime}(1)=\int_{0}^{+\infty} \frac{Q_{k-1}(1 ; t x)}{Q_{k}(1, t x)} t x f(x)
$$


which is strictly finite. Thus, the derivative transformation of $N, N^{\prime}$, has the first $k$ moments finite and its pgf verifies that

$$
h_{N^{\prime}}(s)=\frac{\int_{0}^{+\infty} \frac{Q_{k-1}(s ; t x)}{Q_{k}(1 ; t x)} \operatorname{txf}(x) d x}{\int_{0}^{+\infty} \frac{Q_{k-1}(1 ; t x)}{Q_{k}(1 ; t x)} \operatorname{txf}(x) d x}=\int_{0}^{+\infty} \frac{Q_{k-1}(s ; t x)}{Q_{k-1}(1 ; t x)} g(x) d x
$$

where

$$
g(x)=\frac{Q_{k-1}(1 ; t x)}{Q_{k}(1 ; t x)} t x f(x)\left[\int_{0}^{+\infty} \frac{Q_{k-1}(1 ; t x)}{Q_{k}(1 ; t x)} t x f(x) d x\right]^{-1} .
$$

Given that $g(x)$ is a probability density function on the positive real line, $N^{\prime}$ has a M- $(k-1) \mathrm{TP}$ distribution.

To prove the reverse implication, let us assume that $N^{\prime}$ has a M- $(k-1)$ TP distribution with the first $k$ moments finite, and that it is the derivative transformation of $N$ with pgf $h_{N}(s)$.

Given that $N^{\prime}$ is a mixture of Poisson distributions left-truncated at $k-1$, there exists a constant value $t$ and a non-negative r.v. $\Gamma$ with probability density function $f(x)$ and mean equal to one such that $\lambda=t \Gamma$ and

$$
h_{N^{\prime}}(s)=\int_{0}^{+\infty} \frac{Q_{k-1}(s ; t x)}{Q_{k-1}(1 ; t x)} f(x) d x .
$$

Integrating both sides in $[0, s]$ where $s$ is a positive real number, one has that

$$
\int_{0}^{s} h_{N^{\prime}}(r) d r=\int_{0}^{s}\left[\int_{0}^{+\infty} \frac{Q_{k-1}(r ; t x)}{Q_{k-1}(1 ; t x)} f(x) d x\right] d r
$$

Moreover, taking on account that $N^{\prime}$ is the derivative transformation of $N$ one has that

$$
\int_{0}^{s} h_{N^{\prime}}(r) d r=\frac{h_{N}(s)}{h_{N}^{\prime}(1)}
$$

and given that $h_{N}(1)=1$, because it is a pgf, by (40) and (39) one also has that

$$
\frac{1}{h_{N}^{\prime}(1)}=\int_{0}^{1} h_{M}(r) d r=\int_{0}^{1}\left[\int_{0}^{+\infty} \frac{Q_{k-1}(r ; t x)}{Q_{k-1}(1 ; t x)} f(x) d x\right] d r .
$$

Then, by (39), by (41), by

$$
\int_{0}^{s} Q_{k-1}(r ; t x) d r=\frac{1}{t x} Q_{k}(s ; t x)
$$


and by Fubini's theorem one has that

$$
\begin{aligned}
h_{N}(s) & =\frac{\int_{0}^{s}\left[\int_{0}^{+\infty} \frac{Q_{k-1}(r ; t x)}{Q_{k-1}(1 ; t x)} f(x) d x\right] d r}{\int_{0}^{1}\left[\int_{0}^{+\infty} \frac{Q_{k-1}(r ; t x)}{Q_{k-1}(1 ; t x)} f(x) d x\right] d r}=\frac{\int_{0}^{+\infty}\left[\frac{\int_{0}^{s} Q_{k-1}(r ; t x) d r}{Q_{k-1}(1 ; t x)}\right] f(x) d x}{\int_{0}^{+\infty}\left[\frac{\int_{0}^{1} Q_{k-1}(r ; t x) d r}{Q_{k-1}(1 ; t x)}\right] f(x) d x} \\
& =\int_{0}^{+\infty} \frac{Q_{k}(s ; t x)}{Q_{k}(1, t x)} \frac{\frac{Q_{k}(1 ; t x)}{t x Q_{k-1}(1 ; t x)} f(x)}{\int_{0}^{+\infty} \frac{Q_{k}(1 ; t x)}{t x Q_{k-1}(1 ; t x)} f(x) d x} d x,
\end{aligned}
$$

and given that

$$
\frac{\frac{Q_{k}(1 ; t x)}{t x Q_{k-1}(1 ; t x)} f(x)}{\int_{0}^{+\infty} \frac{Q_{k}(1 ; t x)}{t x Q_{k-1}(1 ; t x)} f(x) d x}
$$

is a density function on the positive real line, by (38) one has that $N$ has a M- $k \mathrm{TP}$ distribution, with finite first $k+1$ moments.

Proof of Theorem 3: By (4), (5) and (38) it follows that the pgf of a M- $k$ TP distribution must verify the four conditions of the theorem.

The reverse implication is proved by induction. Assuming that $h(s)$ is a function verifying the four conditions of the theorem for $k=0$ then, by Theorem 2 of Valero et al. (2010), $h(s)$ is the pgf of a mixture of zero-truncated Poisson distributions with the first moment finite. Furthermore, if $h(s)$ verifies the conditions of the theorem for $k=k_{0}+1$, then the function $h_{M}(s)=h^{\prime}(s) / h^{\prime}(1)$ verifies the conditons of the theorem for $k=k_{0}$ and hence, by the induction assumption, $h_{M}(s)$ is the pgf of a M- $k_{0} \mathrm{TP}$ distribution with the first $k_{0}+1$ moments finite. Hence, $h(s)$ is the pgf of a M- $\left(k_{0}+1\right) \mathrm{TP}$ distribution because of Proposition 2.

Proof of Lemma 2: By (2) one has that if $h(s)$ is the pgf of the left-truncation at $k$ of a mixed Poisson r.v. $N$ with $\operatorname{pgf} h_{N}(s)$, then the derivative transformation of $h(s)$ is equal to

$$
\frac{h^{\prime}(s)}{h^{\prime}(1)}=\frac{\frac{h_{N}^{\prime}(s)}{h_{N}^{\prime}(1)}-\sum_{j=1}^{k} j \frac{P(N=j)}{h_{N}^{\prime}(1)} s^{j-1}}{1-\sum_{j=1}^{k} j \frac{P(N=j)}{h_{N}^{\prime}(1)}}=\frac{\frac{h_{N}^{\prime}(s)}{h_{N}^{\prime}(1)}-\sum_{j=0}^{k-1}(j+1) \frac{P(N=j+1)}{h_{N}^{\prime}(1)} s^{j}}{1-\sum_{j=0}^{k-1}(j+1) \frac{P(N=j+1)}{h_{N}^{\prime}(1)}} .
$$

Given that the derivative transformation of $N$ verifies that

$$
P\left(N^{\prime}=j\right)=\frac{(j+1) P(N=j+1)}{h_{N}^{\prime}(1)},
$$

by (42) $h^{\prime}(s) / h^{\prime}(1)$ is also the pgf of the $k-1$ truncation of $N^{\prime}$.

Proof of Proposition 3: By definition, $N$ follows a $k \mathrm{~T}-\mathrm{M}-i \mathrm{TP}$ distribution if, and only if, it is the left-truncation at $k$ of a r.v. $N^{*}$ which is M- $i$ TP distributed. Applying Lemma 2 one has that the derivative transformation of $N$ is equal to the left-truncation at $k-1$ of the derivative transformation of $N^{*}$, but by Proposition 2, the derivative transformation of $N^{*}$ is M- $(i-1) \mathrm{TP}$ distributed, which proves the result. 
Proof of Theorem 4: Let us consider $k$ fixed. First we prove that $k \mathrm{~T}-\mathrm{MP} \subset k \mathrm{~T}$ M-0TP. Given $h(s)$ a pgf of a MP distribution, it is straightforward to check that its left-truncation at $k$ is equal to the left-truncation at $k$ of its zero-truncation. To prove that $k \mathrm{~T}-\mathrm{M}-i \mathrm{TP}$ is included in $k \mathrm{~T}-\mathrm{M}-(i+1) \mathrm{TP}$ for a given $0 \leq i<k-1$, note that if $N$, with pgf $h_{N}(s)$, follows a $k \mathrm{~T}-\mathrm{M}-i \mathrm{TP}$ distribution, by Corollary 4 one has that the normalization of $h_{N}^{(i+1)}(s)$ is the pgf of a $(k-i-1)$ T-MP distribution. Applying Corollary 1 to $h_{N}^{(i+1)}(s)$ one has that the normalization of $h_{N}^{i+2)}(s)$ is the pgf of a $(k-(i+1)-1) \mathrm{T}-\mathrm{MP}$ distribution, and by using Corollary 4 on $h_{N}^{i+2)}(s)$ one has that $N$ is $k \mathrm{~T}-\mathrm{M}-(i+1) \mathrm{TP}$ distributed.

Proof of Proposition 5: The function $h_{M}(s)=s^{k+1} h_{N}(s)$ verifies conditions $a), b)$ and $c$ ) of theorems 1 and 3. Therefore it is enough to check that if $h_{M}(s)$ satisfies (16), it does not satisfy condition $d$ ). Given that $N$ is mixed Poisson distributed, by Proposition 1 one has that $\epsilon \in[0,1)$. Next, the cases $\epsilon=0$ and $\epsilon \neq 0$ are distinguished.

If $\epsilon=0$, the limit of $h_{M}(s)$ at $-\infty$ is equal to zero by condition (16). Given that, by condition $a), h_{M}(s)$ and their first $k$ derivatives evaluated at zero are all equal to zero, applying the mean value theorem to the function and to their first $k$ derivatives, one has that for $i=1 \cdots k+1$ there exists $s_{0}^{i}<0$ such that $h_{M}^{(i)}\left(s_{0}^{i}\right)=0$. Consequently, $h_{M}^{(k+1)}(s)$ takes the value zero for at least a point in $(-\infty, 0)$ and condition $d)$ is not verifyed.

If $\epsilon>0$, then $N=\epsilon+(1-\epsilon) N^{*}$ where $N^{*}$ is mixed Poisson distributed with limit zero at $\infty$. Given that $N$ verifies (16) if, and only if, $N^{*}$ verifies the same condition with $\epsilon=0$, by condition $a$ ) of Theorems 1 and 3 one has that there exists a value $s_{0}<0$ such that $h_{N^{*}}^{k+2)}(s)<0$. And given that for $n \geq 2$ the signs of $h_{N}^{n)}(s)$ and of $h_{N^{*}}^{n)}(s)$ are the same, it follows that $h_{M}(s)$ does not verify condition $d$ ) and, as a consequence, $M$ is neither $k \mathrm{~T}$-MP distributed nor M- $k \mathrm{TP}$ distributed.

Proof of Proposition 6: Consider $N^{*}$ to be the r.v. with pgf equal to the normalization of the $(k+1)$-th derivative of $h_{N}(s)$,

$$
h_{N^{*}}(s)=\frac{h_{N}^{(k+1)}(s)}{h_{N}^{(k+1)}(1)} .
$$

By Proposition $1, N^{*}$ is a Poisson mixture that verifies (16) with $\epsilon=0$, and that has $M$ as a shifted version of order $k+1$. Thus, the result is a consequence of Proposition 5.

Acknowledgements: This work was funded in part by grants No. TIN200914560-C03-03, MTM2006-09920 and MTM2010-14887 of the Ministerio de Ciencia y Tecnologia of Spain and by Grant No. SGR-1187 of the Generalitat de Catalunya. M. Pérez-Casany wishes to thank the Centre de Recerca Matemàtica, Bellaterra, Spain, for hosting her during part of her sabbatical in 2010. 


\title{
REFERENCES
}

Böhning D and Kuhnert R (2006). Equivalence of Truncated Count Mixture Distributions and Mixtures of Truncated Count Distributions, Biometrics, 62, 1207-1215.

Courant R and John F (1965). Introduction to Calculus and Analysis, Vol. I, Interscience Publishers.

Feller W (1971). An Introduction to Probability Theory and Its Applications, Vol. II Second Edition, N. Y. Wiley.

Hougaard P, Ting Lee M-L and Whitmore GA (1997) Analysis of overdispersed count data by mixtures of Poisson variables and Poisson processes Biometrics, $53(4)$,

Johnson NL, Kemp AW and Kotz S(2005). Univariate Discrete Distributions (Third Edition), Wiley Series in Probability and Mathematical Statistics, Third Edition.

Puig P and Valero J (2006). Count data distributions: Some characterizations with applications. Journal of the American Statistical Association, 101, 332-340. Puri PS and Goldie CM (1979). Poisson mixtures and quasi-infinite divisibility of distributions, Journal of Applied Probability 16, 138-153.

Sichel, HS (1975). On a distribution law for words frequencies, Journal of the American Statistical Association, 70, 542-547.

Sichel, HS (1997). Modelling species-abundance frequencies and speciesindividual functions with the generalized inverse Gaussian-Poisson distribution. South African Statistical Journal, 31, 13-37.

Valero J, Ginebra, J and Pérez-Casany M (2010). Extended truncated TweediePoisson model. Manuscript submitted for publication.

Valero J, Pérez-Casany, M, and Ginebra, J (2010). On Zero-Truncating and Mixing Poisson Distributions. Advances in Applied Probability. 42(4),

\author{
JORDI VALERO \\ Departament de Matemàtica Aplicada 3 \\ ESAB, EDIFICI ESAB D4 064 \\ Avda del Canal Olímpic, S/N \\ Universitat Politècnica de Catalunya \\ 08860 CASTELLDEFELS \\ E-mail address: jordi.valero@upc.edu
}

Marta Pérez-Casany

Departament de Matemàtica Aplicada 2 I DAMA-UPC

EDIFICI OMEGA, JORDi Girona 1-3

Universitat Politècnica de CATAlunya

08034 Barcelona, SPAin

E-mail address: marta.perez@upc.edu 
Josep Ginebra

Departament D'Estadística i InVEstigació Operativa

E.T.S.E.I.B., Avda Diagonal 647, 6A. Planta

Universitat Politècnica de Catalunya

08028 BARCELONA, SPAIN

E-mail address: josep.ginebra@upc.edu 